\title{
An Analytical Study of Knowledge Management Softwares
}

\author{
Dipali P. Meher \\ Research Scholar \\ IMED, Bharati Vidyapeeth, \\ Pune 38
}

\author{
Nilesh Mahajan, PhD \\ Professor \\ IMED, Bharti Vidyapeeth, Pune \\ 38
}

\author{
Pallawi Bulakh, PhD \\ Assistant Professor \\ Modern College of Arts, \\ Science and Commerce, \\ Ganeshkhind, Pune 16
}

\begin{abstract}
The important characteristic of information processing system is its search ability, and should be available at any time anywhere. In 21 st century, knowledge management is an important part of an organization. All organizations, irrespective of their type, are thinking of availability of data, information and knowledge. The real time access to any information which exists in organization is nothing but knowledge on which strategic decisions are based. There are many software's in real life which do knowledge management of an organization. Author has studied 72 knowledge management software's on attributes like advance search, collaboration, content management, data management, discussion boards, document management, full text search, guided problem solving, self service portal, self learning and tried to find out some relationship in these attributes.
\end{abstract}

\section{Keywords}

Knowledge, knowledge management, knowledge management software's

\section{INTRODUCTION}

Knowledge is present in thought and in concept of every individual. It resides in brain of individual and it is used in organizations processes, products, services, systems and outcomes. Knowledge is nothing but result of learning. It comes after data and information. Actually knowledge is authenticated information and thought to be true. So to value it should be stored, tested, focused, shared, used and maintained.

Knowledge management is used in companies to help companies create, share and use knowledge effectively. It is systematic management of vital knowledge. So it is a process of capturing knowledge, understanding knowledge by using information technology systems in order to maintain reuse and deploy that knowledge in order to achieve organization's goals. Knowledge management systems are generally IT based systems used for managing knowledge in organization.

Knowledge management refers to the process of creating, sharing, using, managing information and knowledge of an organization and within the organization. It is always a multidisciplinary approach which is used by any organization to achieve its goal. Many large and small scale organizations are applying knowledge management as a part of their business strategy, information technology and human resource management. Knowledge management typically focuses on organizational objectives, innovations, competitive advantages through lessons learned. Knowledge management is always enabler of organizations learning.

\section{KNOWLEDGE MANAGEMENT SOFTWARE'S}

Knowledge Management is an important tool for any organizations sustainable growth and management from all views [1]. Knowledge Management is always playing fundamental role in any research organization. As in academic organizations research has also become an important criterion to be considered for its valuation and accreditation [2].Many academic organizations receive special status based on their research because their outcome is only knowledge. So to store this research data there are many techniques and tools and methods are used. Many authors have already studied these techniques such as Brainstorming, Learning and Idea Capture

Peer Assist, Learning Reviews, After Action Review,

Storytelling, Collaborative Physical Workspace, APO Knowledge Management Assessment Tool, Knowledge Café, Communities of Practice, Taxonomy, Document Libraries Leading to a Document Management System, Knowledge Bases (Wikis, etc.), Blogs, Social Network Services, Voice and Voice-over-Internet Protocol (VOIP), Advanced Search Tools, Building Knowledge Clusters, Expertise Locator / Who's Who, Collaborative Virtual Workspaces, Knowledge Portal, Video Sharing [3],case based reasoning system, Group Decision Support System, semantic search engines and link machines [4]. Many organizations have their own knowledge base and proper Knowledge Management tools techniques and methods with corresponding value indicators [5].

In any area of research knowledge management is important. There are so many readymade software's available for it

Knowledge management systems: they are IT based systems and designed particularly to promote the creation and sharing of information and knowledge by using various tools and technologies so that information can be turned into knowledge. A Knowledge Management System can be used to help to increase business, staff performance, client satisfaction, and quality of service and product that organization provides [6].

\subsection{Advantages of Using Knowledge Management Software's:}

1) It creates knowledge base for an organization

2) They easily provide support to new technologies

3) They capture knowledge for future use.

4) Due to existing knowledge base employee can find any information at any time

5) Create knowledge base articles

6) Reduces information technology costs without compromising internal and external users. 
7) Improves communication between organizational staff

8) Delivers better measurement and accountability

9) It provides reusability of resources and avoids duplication of information which increases employees confidence

10) Due to different access rights information is tracing becomes easy

11) Online suggestion can be received through feedback system

12) It provides formal and informal training in single eLearning platform

13) New Employees can be easily become familiar with organization and its structures and working environment.

14) It provides more explicit knowledge such as success stories rather than tacit knowledge.

15) Data resources become more discoverable and reusable.[7]

16) Last but not least such systems improves decision making.[8]

17) It enhances team coordination and collaboration

18) Improves the flow of knowledge

19) Helps an organization to grow more network connections, more knowledge nodes

20) Provides better knowledge transfer which occur in execution of projects.

\subsection{Knowledge Management Technologies}

They can be categorized into groupware, workflow, content/ document management systems, enterprise portals, eLearning, scheduling and planning, Telepresence [9].

1) Groupware: It is generally application software which is designed to help people at remote locations who are involved in common task to achieve their goals. They consist of calendar, electronic meetings, collective wittings, shared database access with all persons can see the information's shared with others and all activities.[10]

2) Workflow: Such tools allow the representation of processes which do creation, maintenance and use of organizational knowledge. It also involves all the processes to create forms.

3) Content/Document management: they are the software's that automate process of creating web document and web contents.

4) Enterprise Portals: it is nothing but website that shows collective information about entire organization and its processes to peoples such as project teams.

5) ELearning: it allows organization to create customized training and education software which includes lesson plans, progress monitoring and online teaching.

6) Scheduling and Planning: automatic schedule creation and maintenance i.e. through Microsoft Outlook. And for planning Microsoft Project.
7) Telepresence: it is virtual face to face meetings at remote locations. A common known term is videoconferencing.

8) Apart from above many artificial intelligence tools and software's, simulation tools, semantic networks are available as knowledge sharing tools.

\subsection{Analysis of Knowledge Management Open Source Software's}

Total 72 Software's of Knowledge Sharing in Knowledge Management are studied on the basis of following attributes. If software supports the feature then its value for that cell is 1 otherwise 0 .

- Advance Search $\left(\mathrm{A}_{1}\right)$

- Cataloging/Categorization $\left(\mathrm{A}_{2}\right)$

- Collaboration $\left(\mathrm{A}_{3}\right)$

- Content Management $\left(\mathrm{A}_{4}\right)$

- Data Management $\left(\mathrm{A}_{5}\right)$

- Discussion Boards $\left(\mathrm{A}_{6}\right)$

- Document Management $\left(\mathrm{A}_{7}\right)$

- $\operatorname{FAQ}\left(\mathrm{A}_{8}\right)$

- Full Text Search $\left(\mathrm{A}_{9}\right)$

- $\quad$ Guided Problem Solving $\left(\mathrm{A}_{10}\right)$

- $\quad$ Self Service Portal $\left(\mathrm{A}_{11}\right)$

- $\quad$ Self Learning $\left(\mathrm{A}_{12}\right)$

The database is stored in excel is attached at Annexure 1 (Table 1)

Graph 1 shows the number of software's supporting selection criterion

Hypothesis: $\mathrm{H}_{0}$ : Attribute $\mathrm{A}_{1}, \mathrm{~A}_{2}$ are independent.

The database is created and then test statistics (chi square test) is applied for it. Please refer Annexure 2 (Table 2).

$\chi^{2}=\mathrm{N}(\mathrm{ad}-\mathrm{bc})^{2} /(\mathrm{a}+\mathrm{b})(\mathrm{a}+\mathrm{c})(\mathrm{b}+\mathrm{d})(\mathrm{c}+\mathrm{d})$

Level of significance is $\alpha=0.05$

After applying test statistics -chi square test is applied to see whether the combinations of attributes are accepted or rejects. If value of ts> 3.481 then combination those attribute is rejected otherwise it is accepted. The final result shows whether the attribute are dependent or not. Means if it rejected then they are dependent otherwise not. Annexure 3 (Table 3) shows the answer of chi-square test and its values. Annexure 4 (Table 4) shows final dependent attributes.

Graph 2 shows the combination of attributes which are commonly considered together in all knowledge management software's. The attributes advance search and cataloging and FAQ and guided problem solving are strongly correlated with each other.

\section{CONCLUSION}

In this paper authors have studied various knowledge management software's according some attributes. The study have undertaken for initiative for creation of knowledge sharing software for academic institute. To create such customized software's which attributes are considered first and then later is identified which will be helpful for creation of any knowledge sharing software for academic institute. 
Study shows that attributes dependency (please refer Table3 and Table 4) of already available knowledge management software's. In future authors want to prepare knowledge management framework for knowledge sharing for research in academic institute to avoid knowledge loss across different academic institutions by considering above attributes in priority.

\section{REFERENCES}

[1] Omotayo, Funmilola Olubunmi, "Knowledge Management as an important tool in Organizational Management: A Review of Literature" (2015). Library Philosophy and Practice (e-journal). Paper 1238.

[2]Ermine, J. "Methods and Tools for Knowledge Management in Research Centers" Electronic Journal of Knowledge Management Volume 8 Issue 3 (pp293 306), available online at www.ejkm com

[3]Ronald Young,(2010), "Knowledge Management Tools and Techniques Manual", Asian Productivity Organization.

[4] Syed Ghani, (2009),"Knowledge Management: Tools and Techniques", DESIDOC Journal of Library \& Information Technology, Vol. 29, No. 6, pp. 33-38
[5]Yang Xu, Alain Bernard, Nicolas Perry, Lian Lian. Managing knowledge management tools: a systematic classification and comparison. The 2011 International Conference on Management and Service Science (MASS), Aug 2011, China. IEEE - Institute of Electrical and Electronics Engineers, pp.1-4, 2011.

[6]Craciun Elisei,(2014), "Benefits of a Knowledge Management System Within an Organization"

[7] Kane Jim, (2014) Life Sciences, Information Management, "8 Benefits of having knowledge management strategy", Also available at http://www.consultparagon.com/blog/8 benefits-of-knowledge-management.

[8] KiKM,(2005), "WHAT ARE THE BENEFITS OF KM?"

[9] Frost Alon,(2017), "Knowledge Management Tools”, KM from $\mathrm{A}$ to $\mathrm{Z}$

[10]TechTarget Groupware, Also available at "http://searchdomino.techtarget.com/definition/groupwar e

\section{APPENDIX}

\section{Annexure 1}

Table 1: Data of software's

\begin{tabular}{|c|c|c|c|c|c|c|c|c|c|c|c|c|c|}
\hline Sr.No. & Software Name & $\mathbf{A}_{1}$ & $\mathbf{A}_{2}$ & $\mathbf{A}_{3}$ & $\mathbf{A}_{4}$ & $\mathbf{A}_{5}$ & $\mathbf{A}_{6}$ & $\mathbf{A}_{7}$ & $\mathbf{A}_{8}$ & $\mathbf{A}_{9}$ & $\mathbf{A}_{10}$ & $\mathbf{A}_{11}$ & $\mathbf{A}_{12}$ \\
\hline 1 & myTips & 0 & 0 & 1 & 0 & 0 & 0 & 0 & 1 & 0 & 1 & 1 & 0 \\
\hline 2 & Astute Knowledge & 0 & 0 & 0 & 1 & 1 & 0 & 1 & 1 & 0 & 1 & 0 & 1 \\
\hline 3 & Berkeley Publisher & 0 & 0 & 1 & 0 & 0 & 0 & 1 & 0 & 0 & 1 & 1 & 0 \\
\hline 4 & ManIPulate & 0 & 0 & 0 & 0 & 1 & 0 & 1 & 0 & 1 & 0 & 0 & 1 \\
\hline 5 & Querybot & 0 & 0 & 0 & 0 & 1 & 0 & 0 & 1 & 1 & 0 & 0 & 0 \\
\hline 6 & Correlate K-Map & 0 & 1 & 1 & 0 & 1 & 0 & 1 & 0 & 0 & 0 & 0 & 1 \\
\hline 7 & Unity & 1 & 0 & 1 & 1 & 0 & 0 & 1 & 1 & 1 & 0 & 1 & 0 \\
\hline 8 & FirmCover & 1 & 0 & 1 & 0 & 0 & 0 & 1 & 0 & 1 & 0 & 0 & 0 \\
\hline 9 & APTEAN Knowledge Management & 1 & 0 & 0 & 0 & 0 & 0 & 1 & 1 & 0 & 0 & 1 & 1 \\
\hline 10 & GemShelf & 1 & 0 & 1 & 1 & 0 & 0 & 1 & 0 & 1 & 0 & 1 & 0 \\
\hline 11 & Haydle & 1 & 0 & 1 & 0 & 0 & 0 & 0 & 0 & 1 & 0 & 1 & 1 \\
\hline 12 & Helprace & 1 & 0 & 1 & 0 & 1 & 1 & 1 & 0 & 1 & 1 & 1 & 0 \\
\hline 13 & Indexedmind & 1 & 0 & 1 & 0 & 0 & 1 & 0 & 0 & 0 & 0 & 0 & 0 \\
\hline 14 & IntelliResponse Virtual Agent & 1 & 0 & 0 & 0 & 0 & 0 & 0 & 1 & 1 & 1 & 1 & 1 \\
\hline 15 & LuitDox Platinum & 1 & 0 & 0 & 0 & 0 & 1 & 1 & 0 & 0 & 0 & 0 & 0 \\
\hline 16 & PeerAware & 1 & 0 & 1 & 0 & 0 & 0 & 0 & 0 & 1 & 0 & 1 & 1 \\
\hline 17 & Union Square & 1 & 0 & 1 & 0 & 1 & 1 & 1 & 0 & 1 & 0 & 0 & 0 \\
\hline 18 & Confluence & 1 & 1 & 1 & 0 & 0 & 1 & 1 & 1 & 1 & 0 & 1 & 1 \\
\hline 19 & Freshdesk & 1 & 1 & 0 & 0 & 0 & 1 & 0 & 1 & 1 & 1 & 1 & 1 \\
\hline 20 & eXo Platform & 1 & 1 & 1 & 0 & 0 & 1 & 1 & 0 & 1 & 0 & 0 & 0 \\
\hline 21 & Zendesk & 1 & 1 & 0 & 0 & 1 & 1 & 1 & 1 & 1 & 0 & 1 & 1 \\
\hline 22 & Bitrix 24 & 1 & 1 & 1 & 0 & 1 & 1 & 1 & 1 & 1 & 1 & 1 & 1 \\
\hline 23 & Novo Knowledge Base Software & 1 & 1 & 0 & 0 & 0 & 1 & 1 & 0 & 1 & 0 & 1 & 1 \\
\hline 24 & FuzeDigital & 1 & 1 & 1 & 0 & 1 & 1 & 1 & 1 & 1 & 1 & 1 & 1 \\
\hline 25 & PHPKB & 1 & 1 & 1 & 1 & 1 & 0 & 1 & 1 & 1 & 0 & 1 & 1 \\
\hline
\end{tabular}




\begin{tabular}{|c|c|c|c|c|c|c|c|c|c|c|c|c|c|}
\hline 26 & AnswerHub & 1 & 1 & 1 & 1 & 0 & 1 & 1 & 1 & 1 & 1 & 1 & 1 \\
\hline 27 & Intelligence2day & 1 & 1 & 1 & 1 & 1 & 1 & 1 & 0 & 1 & 0 & 0 & 0 \\
\hline 28 & Lessons Learned Server & 1 & 1 & 1 & 1 & 1 & 0 & 1 & 0 & 1 & 0 & 0 & 1 \\
\hline 29 & Qiqqa & 1 & 1 & 1 & 0 & 1 & 0 & 0 & 1 & 1 & 0 & 1 & 1 \\
\hline 30 & Unified Knowledge Platform & 1 & 1 & 1 & 0 & 1 & 1 & 1 & 1 & 1 & 1 & 1 & 1 \\
\hline 31 & Qbeeko & 1 & 1 & 1 & 1 & 1 & 1 & 0 & 1 & 1 & 1 & 1 & 1 \\
\hline 32 & AGI Self Service & 1 & 1 & 1 & 0 & 1 & 0 & 0 & 1 & 1 & 1 & 1 & 1 \\
\hline 33 & Assyst & 1 & 1 & 1 & 1 & 1 & 1 & 1 & 1 & 1 & 1 & 1 & 1 \\
\hline 34 & Auros & 1 & 1 & 1 & 1 & 1 & 1 & 1 & 1 & 1 & 1 & 1 & 1 \\
\hline 35 & BIKS & 1 & 1 & 1 & 0 & 1 & 1 & 1 & 1 & 1 & 1 & 1 & 1 \\
\hline 36 & BoxesOS & 1 & 1 & 1 & 0 & 1 & 1 & 1 & 1 & 0 & 1 & 1 & 0 \\
\hline 37 & ComAround Zero & 1 & 1 & 0 & 1 & 1 & 0 & 1 & 1 & 1 & 1 & 1 & 1 \\
\hline 38 & Comm100 Knowledge Base & 1 & 1 & 0 & 1 & 0 & 1 & 1 & 0 & 1 & 1 & 1 & 1 \\
\hline 39 & CommonSense & 1 & 1 & 1 & 0 & 0 & 1 & 1 & 1 & 1 & 1 & 1 & 1 \\
\hline 40 & Connotate & 1 & 1 & 1 & 0 & 1 & 0 & 0 & 0 & 1 & 0 & 1 & 1 \\
\hline 41 & digi-libris Reader & 1 & 1 & 1 & 1 & 1 & 0 & 1 & 0 & 0 & 0 & 0 & 0 \\
\hline 42 & DoyleSoft Knowledge Base & 1 & 1 & 1 & 0 & 1 & 0 & 1 & 0 & 1 & 1 & 1 & 1 \\
\hline 43 & eKMS & 1 & 1 & 1 & 0 & 0 & 0 & 1 & 0 & 0 & 1 & 1 & 1 \\
\hline 44 & Enterprise Wiki & 1 & 1 & 1 & 0 & 1 & 0 & 1 & 1 & 0 & 1 & 1 & 0 \\
\hline 45 & Guru & 1 & 1 & 1 & 1 & 1 & 1 & 1 & 0 & 0 & 0 & 1 & 1 \\
\hline 46 & HelpConsole & 1 & 1 & 1 & 1 & 1 & 0 & 1 & 1 & 1 & 0 & 1 & 1 \\
\hline 47 & Heroic Knowledge Base & 1 & 1 & 1 & 0 & 1 & 0 & 1 & 1 & 1 & 0 & 0 & 1 \\
\hline 48 & Hivemind & 1 & 1 & 1 & 0 & 0 & 1 & 1 & 0 & 1 & 0 & 1 & 0 \\
\hline 49 & Inmagic Presto & 1 & 1 & 0 & 0 & 1 & 0 & 1 & 1 & 0 & 0 & 1 & 0 \\
\hline 50 & Interspire Knowledge Manager & 1 & 1 & 1 & 0 & 1 & 0 & 1 & 1 & 1 & 1 & 1 & 0 \\
\hline 51 & Knowledge Management Suite & 1 & 1 & 1 & 0 & 1 & 0 & 1 & 1 & 1 & 1 & 1 & 0 \\
\hline 52 & KnowledgeBase & 1 & 1 & 1 & 0 & 1 & 1 & 0 & 0 & 0 & 0 & 0 & 0 \\
\hline 53 & Knowledgebase Manager Pro & 1 & 1 & 1 & 1 & 1 & 1 & 1 & 1 & 1 & 0 & 1 & 0 \\
\hline 54 & Kreeo & 1 & 1 & 1 & 1 & 1 & 1 & 1 & 1 & 1 & 0 & 1 & 1 \\
\hline 55 & LearnLode & 1 & 1 & 1 & 1 & 0 & 1 & 1 & 1 & 0 & 1 & 0 & 1 \\
\hline 56 & Lumo Flow & 1 & 1 & 1 & 0 & 1 & 1 & 1 & 1 & 1 & 0 & 1 & 1 \\
\hline 57 & OmniknowledgeFAQ & 1 & 1 & 0 & 1 & 0 & 0 & 0 & 1 & 1 & 0 & 1 & 0 \\
\hline 58 & POC Link & 1 & 1 & 0 & 0 & 1 & 0 & 1 & 1 & 1 & 1 & 1 & 1 \\
\hline 59 & Problem Solver & 1 & 1 & 1 & 1 & 0 & 1 & 0 & 1 & 1 & 1 & 1 & 1 \\
\hline 60 & ProProfs Knowledge Base & 1 & 1 & 0 & 1 & 1 & 0 & 1 & 1 & 0 & 0 & 1 & 0 \\
\hline 61 & Question and Answer & 1 & 1 & 1 & 0 & 0 & 1 & 0 & 1 & 1 & 0 & 1 & 1 \\
\hline 62 & ROK'IT & 1 & 1 & 1 & 1 & 1 & 1 & 1 & 1 & 1 & 1 & 0 & 0 \\
\hline 63 & ScreenSteps & 1 & 1 & 1 & 0 & 0 & 0 & 0 & 0 & 1 & 0 & 1 & 1 \\
\hline 64 & Sem.Note & 1 & 1 & 1 & 0 & 0 & 0 & 0 & 0 & 1 & 0 & 0 & 0 \\
\hline 65 & SlimWiki & 1 & 1 & 1 & 0 & 0 & 0 & 1 & 0 & 1 & 0 & 1 & 0 \\
\hline 66 & Social Collaboration & 1 & 1 & 1 & 0 & 1 & 1 & 1 & 1 & 1 & 1 & 1 & 1 \\
\hline 67 & StreetSmarts Knowledge Base & 1 & 1 & 1 & 0 & 1 & 1 & 1 & 1 & 1 & 1 & 1 & 1 \\
\hline 68 & SupportPoint & 1 & 1 & 0 & 0 & 1 & 0 & 1 & 1 & 0 & 1 & 1 & 0 \\
\hline 69 & USU Knowledge Center & 1 & 1 & 1 & 1 & 1 & 1 & 1 & 1 & 1 & 1 & 1 & 1 \\
\hline 70 & WebBoard Knowledge Base & 1 & 1 & 1 & 0 & 1 & 1 & 1 & 1 & 1 & 0 & 0 & 0 \\
\hline 71 & WSN Insight & 1 & 1 & 1 & 0 & 0 & 1 & 0 & 0 & 1 & 0 & 1 & 1 \\
\hline 72 & XPERT Knowledge & 1 & 1 & 0 & 0 & 1 & 1 & 1 & 0 & 0 & 0 & 1 & 0 \\
\hline
\end{tabular}


Table 2: Analysis of knowledge management software's based on chi square test

\begin{tabular}{|c|c|c|c|c|c|c|c|c|c|c|}
\hline Combination & $\mathbf{A}$ & b & c & d & $\mathbf{a}+\mathbf{b}$ & $a+c$ & $b+d$ & $c+d$ & ad-bc & ts \\
\hline $\mathrm{A}_{1} \mathrm{~A}_{2}$ & 55 & 11 & 1 & 5 & 66 & 56 & 16 & 6 & 264 & 14.1429 \\
\hline $\mathrm{A}_{1} \mathrm{~A}_{3}$ & 52 & 14 & 3 & 3 & 66 & 55 & 17 & 6 & 114 & 2.5272 \\
\hline $\mathrm{A}_{1} \mathrm{~A}_{4}$ & 22 & 44 & 1 & 5 & 66 & 23 & 49 & 6 & 66 & 0.7028 \\
\hline $\mathrm{A}_{1} \mathrm{~A}_{5}$ & 40 & 26 & 4 & 2 & 66 & 44 & 28 & 6 & -24 & 0.0850 \\
\hline $\mathrm{A}_{1} \mathrm{~A}_{6}$ & 37 & 29 & 0 & 6 & 66 & 37 & 35 & 6 & 222 & 6.9195 \\
\hline $\mathrm{A}_{1} \mathrm{~A}_{7}$ & 50 & 16 & 4 & 2 & 66 & 54 & 18 & 6 & 36 & 0.2424 \\
\hline $\mathrm{A}_{1} \mathrm{~A}_{8}$ & 41 & 25 & 3 & 3 & 66 & 44 & 28 & 6 & 48 & 0.3400 \\
\hline $\mathrm{A}_{1} \mathrm{~A}_{9}$ & 52 & 14 & 2 & 4 & 66 & 54 & 18 & 6 & 180 & 6.0606 \\
\hline $\mathrm{A}_{1} \mathrm{~A}_{10}$ & 29 & 37 & 3 & 3 & 66 & 32 & 40 & 6 & -24 & 0.0818 \\
\hline $\mathrm{A}_{1} \mathrm{~A}_{11}$ & 52 & 14 & 2 & 4 & 66 & 54 & 18 & 6 & 180 & 6.0606 \\
\hline $\mathrm{A}_{1} \mathrm{~A}_{12}$ & 40 & 26 & 3 & 3 & 66 & 43 & 29 & 6 & 42 & 0.2572 \\
\hline $\mathrm{A}_{2} \mathrm{~A}_{3}$ & 45 & 11 & 10 & 6 & 56 & 55 & 17 & 16 & 160 & 2.2002 \\
\hline $\mathrm{A}_{2} \mathrm{~A}_{4}$ & 20 & 36 & 3 & 13 & 56 & 23 & 49 & 16 & 152 & 1.6474 \\
\hline $\mathrm{A}_{2} \mathrm{~A}_{5}$ & 39 & 17 & 5 & 11 & 56 & 44 & 28 & 16 & 344 & 7.7185 \\
\hline $\mathrm{A}_{2} \mathrm{~A}_{6}$ & 33 & 23 & 4 & 12 & 56 & 37 & 35 & 16 & 304 & 5.7346 \\
\hline $\mathrm{A}_{2} \mathrm{~A}_{7}$ & 44 & 12 & 10 & 6 & 56 & 54 & 18 & 16 & 144 & 1.7143 \\
\hline $\mathrm{A}_{2} \mathrm{~A}_{8}$ & 38 & 18 & 6 & 10 & 56 & 44 & 28 & 16 & 272 & 4.8256 \\
\hline $\mathrm{A}_{2} \mathrm{~A}_{9}$ & 44 & 12 & 10 & 6 & 56 & 54 & 18 & 16 & 144 & 1.7143 \\
\hline $\mathrm{A}_{2} \mathrm{~A}_{10}$ & 27 & 29 & 5 & 11 & 56 & 32 & 40 & 16 & 152 & 1.4504 \\
\hline $\mathrm{A}_{2} \mathrm{~A}_{11}$ & 45 & 11 & 9 & 7 & 56 & 54 & 18 & 16 & 216 & 3.8571 \\
\hline $\mathrm{A}_{2} \mathrm{~A}_{12}$ & 37 & 19 & 6 & 10 & 56 & 43 & 29 & 16 & 256 & 4.2232 \\
\hline $\mathrm{A}_{3} \mathrm{~A}_{4}$ & 18 & 37 & 5 & 12 & 55 & 23 & 49 & 17 & 31 & 0.0657 \\
\hline $\mathrm{A}_{3} \mathrm{~A}_{5}$ & 34 & 21 & 10 & 7 & 55 & 44 & 28 & 17 & 28 & 0.0490 \\
\hline $\mathrm{A}_{3} \mathrm{~A}_{6}$ & 31 & 24 & 6 & 11 & 55 & 37 & 35 & 17 & 197 & 2.3077 \\
\hline $\mathrm{A}_{3} \mathrm{~A}_{7}$ & 41 & 14 & 13 & 4 & 55 & 54 & 18 & 17 & -18 & 0.0257 \\
\hline $\mathrm{A}_{3} \mathrm{~A}_{8}$ & 32 & 23 & 12 & 5 & 55 & 44 & 28 & 17 & -116 & 0.8411 \\
\hline $\mathrm{A}_{3} \mathrm{~A}_{9}$ & 44 & 11 & 10 & 7 & 55 & 54 & 18 & 17 & 198 & 3.1059 \\
\hline $\mathrm{A}_{3} \mathrm{~A}_{10}$ & 25 & 30 & 7 & 10 & 55 & 32 & 40 & 17 & 40 & 0.0963 \\
\hline $\mathrm{A}_{3} \mathrm{~A}_{11}$ & 41 & 14 & 13 & 4 & 55 & 54 & 18 & 17 & -18 & 0.0257 \\
\hline $\mathrm{A}_{3} \mathrm{~A}_{12}$ & 33 & 22 & 10 & 7 & 55 & 43 & 29 & 17 & 11 & 0.0075 \\
\hline $\mathrm{A}_{4} \mathrm{~A}_{5}$ & 16 & 7 & 28 & 21 & 23 & 44 & 28 & 49 & 140 & 1.0164 \\
\hline $\mathrm{A}_{4} \mathrm{~A}_{6}$ & 13 & 10 & 24 & 25 & 23 & 37 & 35 & 49 & 85 & 0.3564 \\
\hline $\mathrm{A}_{4} \mathrm{~A}_{7}$ & 20 & 3 & 34 & 15 & 23 & 54 & 18 & 49 & 198 & 2.5768 \\
\hline $\mathrm{A}_{4} \mathrm{~A}_{8}$ & 17 & 6 & 27 & 22 & 23 & 44 & 28 & 49 & 212 & 2.3306 \\
\hline $\mathrm{A}_{4} \mathrm{~A}_{9}$ & 18 & 5 & 36 & 13 & 23 & 54 & 18 & 49 & 54 & 0.1917 \\
\hline $\mathrm{A}_{4} \mathrm{~A}_{10}$ & 11 & 12 & 21 & 28 & 23 & 32 & 40 & 49 & 56 & 0.1565 \\
\hline $\mathrm{A}_{4} \mathrm{~A}_{11}$ & 17 & 6 & 37 & 12 & 23 & 54 & 18 & 49 & -18 & 0.0213 \\
\hline $\mathrm{A}_{4} \mathrm{~A}_{12}$ & 15 & 8 & 28 & 21 & 23 & 43 & 29 & 49 & 91 & 0.4243 \\
\hline $\mathrm{A}_{5} \mathrm{~A}_{6}$ & 23 & 21 & 14 & 14 & 44 & 37 & 35 & 28 & 28 & 0.0354 \\
\hline $\mathrm{A}_{5} \mathrm{~A}_{7}$ & 38 & 6 & 16 & 12 & 44 & 54 & 18 & 28 & 360 & 7.7922 \\
\hline $\mathrm{A}_{5} \mathrm{~A}_{8}$ & 32 & 12 & 12 & 16 & 44 & 44 & 28 & 28 & 368 & 6.4240 \\
\hline $\mathrm{A}_{5} \mathrm{~A}_{9}$ & 33 & 11 & 21 & 7 & 44 & 54 & 18 & 28 & 0 & 0.0000 \\
\hline $\mathrm{A}_{5} \mathrm{~A}_{10}$ & 22 & 22 & 10 & 18 & 44 & 32 & 40 & 28 & 176 & 1.4143 \\
\hline
\end{tabular}




\begin{tabular}{|c|c|c|c|c|c|c|c|c|c|c|}
\hline $\mathrm{A}_{5} \mathrm{~A}_{11}$ & 32 & 12 & 22 & 6 & 44 & 54 & 18 & 28 & -72 & 0.3117 \\
\hline $\mathrm{A}_{5} \mathrm{~A}_{12}$ & 27 & 17 & 16 & 12 & 44 & 43 & 29 & 28 & 52 & 0.1267 \\
\hline $\mathrm{A}_{6} \mathrm{~A}_{7}$ & 30 & 7 & 24 & 11 & 37 & 54 & 18 & 35 & 162 & 1.5012 \\
\hline $\mathrm{A}_{6} \mathrm{~A}_{8}$ & 24 & 13 & 20 & 15 & 37 & 44 & 28 & 35 & 100 & 0.4513 \\
\hline $\mathrm{A}_{6} \mathrm{~A}_{9}$ & 30 & 7 & 24 & 11 & 37 & 54 & 18 & 35 & 162 & 1.5012 \\
\hline $\mathrm{A}_{6} \mathrm{~A}_{10}$ & 19 & 18 & 13 & 22 & 37 & 32 & 40 & 35 & 184 & 1.4706 \\
\hline $\mathrm{A}_{6} \mathrm{~A}_{11}$ & 28 & 9 & 26 & 9 & 37 & 54 & 18 & 35 & 18 & 0.0185 \\
\hline $\mathrm{A}_{6} \mathrm{~A}_{12}$ & 24 & 13 & 19 & 16 & 37 & 43 & 29 & 35 & 137 & 0.8368 \\
\hline $\mathrm{A}_{7} \mathrm{~A}_{8}$ & 34 & 20 & 10 & 8 & 54 & 44 & 28 & 18 & 72 & 0.3117 \\
\hline $\mathrm{A}_{7} \mathrm{~A}_{9}$ & 39 & 15 & 15 & 3 & 54 & 54 & 18 & 18 & -108 & 0.8889 \\
\hline $\mathrm{A}_{7} \mathrm{~A}_{10}$ & 26 & 28 & 6 & 12 & 54 & 32 & 40 & 18 & 144 & 1.2000 \\
\hline $\mathrm{A}_{7} \mathrm{~A}_{11}$ & 40 & 14 & 14 & 4 & 54 & 54 & 18 & 18 & -36 & 0.0988 \\
\hline $\mathrm{A}_{7} \mathrm{~A}_{12}$ & 31 & 23 & 12 & 6 & 54 & 43 & 29 & 18 & -90 & 0.4812 \\
\hline $\mathrm{A}_{8} \mathrm{~A}_{9}$ & 35 & 9 & 19 & 9 & 44 & 54 & 18 & 28 & 144 & 1.2468 \\
\hline $\mathrm{A}_{8} \mathrm{~A}_{10}$ & 27 & 17 & 5 & 23 & 44 & 32 & 40 & 28 & 536 & 13.1172 \\
\hline $\mathrm{A}_{8} \mathrm{~A}_{11}$ & 38 & 6 & 16 & 12 & 44 & 54 & 18 & 28 & 360 & 7.7922 \\
\hline $\mathrm{A}_{8} \mathrm{~A}_{12}$ & 30 & 14 & 13 & 15 & 44 & 43 & 29 & 28 & 268 & 3.3661 \\
\hline $\mathrm{A}_{9} \mathrm{~A}_{10}$ & 24 & 30 & 8 & 10 & 54 & 32 & 40 & 18 & 0 & 0.0000 \\
\hline $\mathrm{A}_{9} \mathrm{~A}_{11}$ & 43 & 11 & 11 & 7 & 54 & 54 & 18 & 18 & 180 & 2.4691 \\
\hline $\mathrm{A}_{9} \mathrm{~A}_{12}$ & 37 & 17 & 6 & 12 & 54 & 43 & 29 & 18 & 342 & 6.9479 \\
\hline $\mathrm{A}_{10} \mathrm{~A}_{11}$ & 29 & 3 & 25 & 15 & 32 & 54 & 18 & 40 & 360 & 7.5000 \\
\hline $\mathrm{A}_{10} \mathrm{~A}_{12}$ & 23 & 9 & 20 & 20 & 32 & 43 & 29 & 40 & 280 & 3.5365 \\
\hline $\mathrm{A}_{11} \mathrm{~A}_{12}$ & 37 & 17 & 6 & 12 & 54 & 43 & 29 & 18 & 342 & 6.9479 \\
\hline & & & & & & & & & & \\
\hline
\end{tabular}

The value of test statistics (i.e. ts)> 3.481 then rejects the hypothesis otherwise accept the hypothesis. If hypothesis is rejected means attributes are dependent on each other. If the hypothesis is accepted then the attributes are independent on each other. So analysis shows following attributes are dependent on each other.

\section{Annexure 3}

Table 3: Analysis of knowledge management software's (for finding dependency)

\begin{tabular}{|c|c|c|l|}
\hline Combination & Accept/Reject & Dependency & \multicolumn{1}{|c|}{ Attributes } \\
\hline $\mathrm{A}_{1} \mathrm{~A}_{2}$ & Reject & Dependent & Advance Search and Cataloging/Categorization \\
\hline $\mathrm{A}_{1} \mathrm{~A}_{6}$ & Reject & Dependent & Advance Search and Discussion boards \\
\hline $\mathrm{A}_{1} \mathrm{~A}_{9}$ & Reject & Dependent & Advance Search and Full Text Search \\
\hline $\mathrm{A}_{1} \mathrm{~A}_{11}$ & Reject & Dependent & Advance search and Self Service Portal \\
\hline $\mathrm{A}_{2} \mathrm{~A}_{5}$ & Reject & Dependent & Cataloging/Categorization and Data Management \\
\hline $\mathrm{A}_{2} \mathrm{~A}_{6}$ & Reject & Dependent & Cataloging/Categorization and Discussion Boards \\
\hline $\mathrm{A}_{2} \mathrm{~A}_{8}$ & Reject & Dependent & Cataloging/Categorization and FAQ \\
\hline $\mathrm{A}_{2} \mathrm{~A}_{11}$ & Reject & Dependent & Cataloging/Categorization and Self Service Portal \\
\hline $\mathrm{A}_{2} \mathrm{~A}_{12}$ & Reject & Dependent & Cataloging/Categorization and Self Learning \\
\hline $\mathrm{A}_{5} \mathrm{~A}_{7}$ & Reject & Dependent & Data Management and Document Management \\
\hline $\mathrm{A}_{5} \mathrm{~A}_{8}$ & Reject & Dependent & Data Management and FAQ \\
\hline $\mathrm{A}_{8} \mathrm{~A}_{10}$ & Reject & Dependent & FAQ and Guided Problem Solving \\
\hline
\end{tabular}




\begin{tabular}{|l|l|l|l|}
$\mathrm{A}_{8} \mathrm{~A}_{11}$ & Reject & Dependent & FAQ and Self Service Portal \\
\hline $\mathrm{A}_{9} \mathrm{~A}_{12}$ & Reject & Dependent & Full Text Search and Self Learning \\
\hline $\mathrm{A}_{10} \mathrm{~A}_{11}$ & Reject & Dependent & Guided Problem Solving and Self Service Portal \\
\hline $\mathrm{A}_{10} \mathrm{~A}_{12}$ & Reject & Dependent & Guided Problem solving and Self Learning \\
\hline $\mathrm{A}_{11} \mathrm{~A}_{12}$ & Reject & Dependent & Self Service Portal and Self Learning \\
\hline
\end{tabular}

\section{Annexure 4}

Table 4: Final dependent attributes

\begin{tabular}{|l|l|}
\hline \multicolumn{1}{|c|}{ Combination } & \multicolumn{1}{c|}{ Attributes } \\
\hline $\mathrm{A}_{1} \mathrm{~A}_{2} \mathrm{~A}_{6} \mathrm{~A}_{9} \mathrm{~A}_{11}$ & $\begin{array}{l}\text { Advance Search depends on Cataloging/Categorization, Discussion boards, Full Text Search, Self } \\
\text { Service Portal }\end{array}$ \\
\hline $\mathrm{A}_{2} \mathrm{~A}_{5} \mathrm{~A}_{6} \mathrm{~A}_{8} \mathrm{~A}_{11} \mathrm{~A}_{12}$ & $\begin{array}{l}\text { Cataloging/Categorization depends on Data Management, Discussion Boards, FAQ, Self Service } \\
\text { Portal, Self Learning }\end{array}$ \\
\hline $\mathrm{A}_{5} \mathrm{~A}_{7} \mathrm{~A}_{8}$ & Data Management depends on Document Management, FAQ \\
\hline $\mathrm{A}_{8} \mathrm{~A}_{10} \mathrm{~A}_{11}$ & FAQ depends on Guided Problem Solving, Self Service Portal \\
\hline $\mathrm{A}_{9} \mathrm{~A}_{12}$ & Full Text Search depends on Self Learning \\
\hline $\mathrm{A}_{10} \mathrm{~A}_{11} \mathrm{~A}_{12}$ & Guided Problem Solving depends on Self Service Portal and Self Learning \\
\hline $\mathrm{A}_{11} \mathrm{~A}_{12}$ & Self Service Portal depends on Self Learning \\
\hline
\end{tabular}

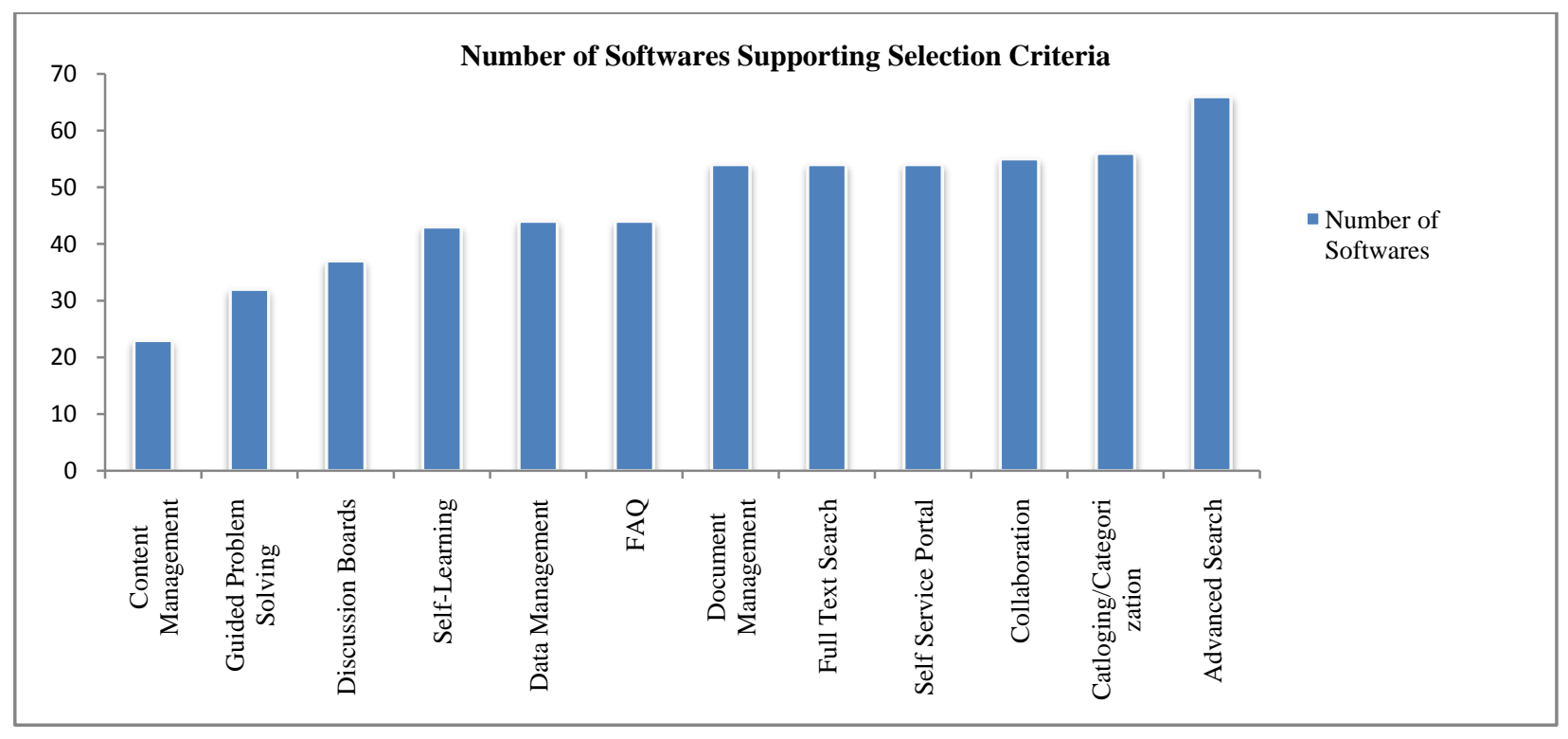

Graph 1: Number of Software's supporting Selection Criteria 


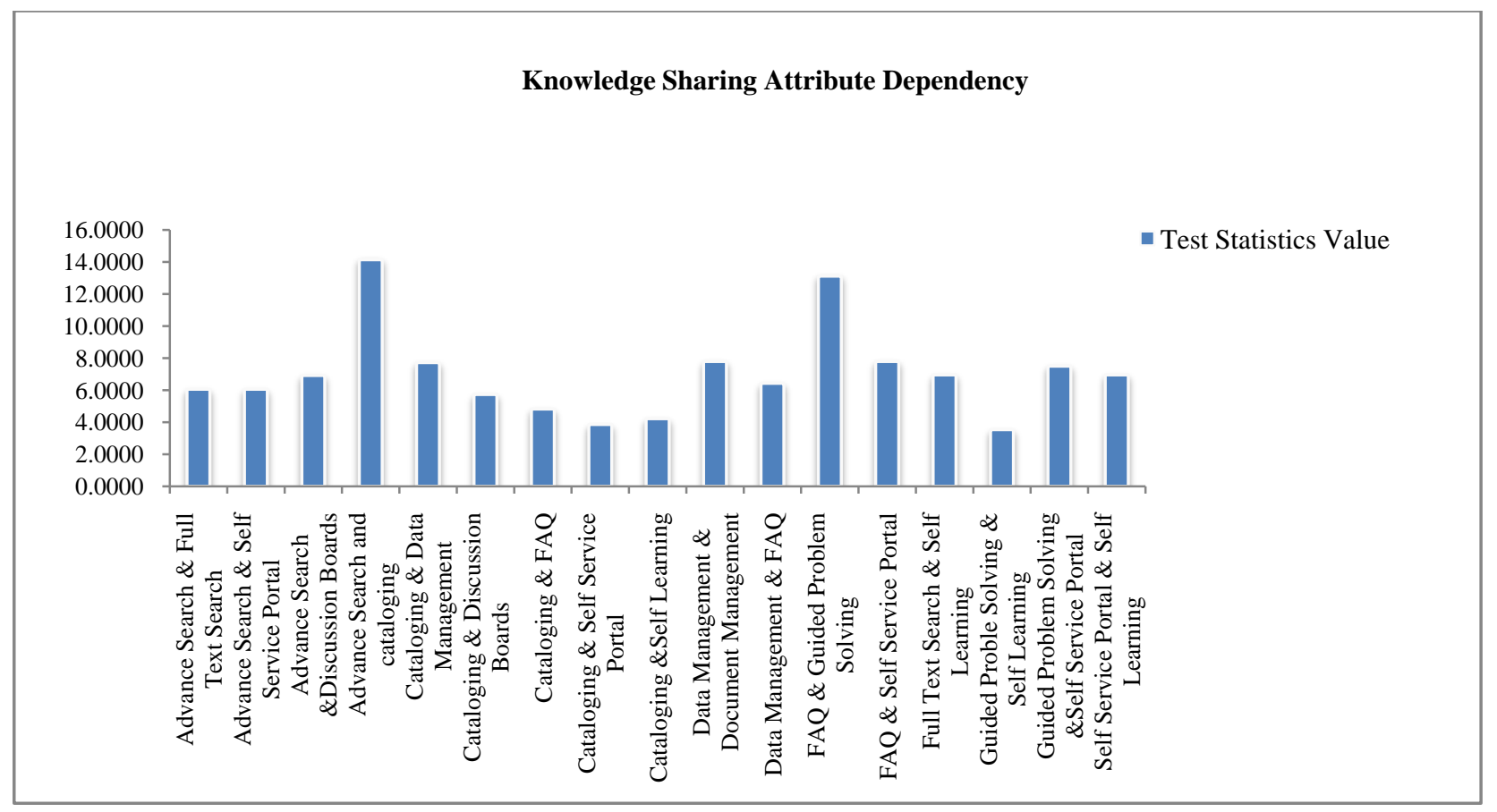

Graph 2: Knowledge Sharing Attribute Dependency 